\title{
ISOMORPHIC EXPONENTIAL WEYL ALGEBRAS
}

\author{
by P. L. ROBINSON
}

(Received 3 June, 1989)

Canonically associated to a real symplectic vector space are several associative algebras. The Weyl algebra (generated by the Heisenberg commutation relations) has been the subject of much study; see [1] for example. The exponential Weyl algebra (generated by the canonical commutation relations in exponential form) has been less well studied; see [8].

It is known that the Weyl algebras associated to symplectic vector spaces of differing dimensions are not isomorphic. This follows from the fact that the Weyl algebra associated to a symplectic vector space of dimension $2 m$ has Krull dimension $m$ (see [6]) and global homological dimension $m$ (see [9]). In this note, we show that the exponential Weyl algebras associated to symplectic vector spaces of differing dimensions are not isomorphic. We accomplish this by a consideration of subalgebras: in fact, we show that the exponential Weyl algebra associated to an $n$-dimensional symplectic vector space has maximal abelian subalgebras generated by $n$ units together with their inverses but has none generated by fewer.

To set the stage, let $(V, \Omega)$ be a real symplectic vector space of dimension $2 m$. The exponential Weyl algebra $A(V, \Omega)$ is the complex associative algebra of all finitelysupported maps from $V$ to $\mathbb{C}$, with pointwise linear structure and with product given by

$$
(\phi . \psi)(v)=\sum_{x+y=v} \varepsilon(x, y) \phi(x) \psi(y)
$$

for $\phi, \psi \in A(V, \Omega)$ and $v \in V$, where

$$
x, y \in V \Rightarrow \varepsilon(x, y)=\exp \{-i \pi \Omega(x, y)\}
$$

for convenience, we define $\omega=\varepsilon^{2}$. Thus, $A(V, \Omega)$ is a twisted group algebra of the additive group $V$, the twist being provided by the cocycle $\varepsilon$; otherwise said, $A(V, \Omega)$ is a crossed product of $\mathbb{C}$ with the group algebra of the discrete group $V$. If for $v \in V$ we define $\delta_{v} \in A(V, \Omega)$ by

$$
\delta_{v}(z)= \begin{cases}1 & \text { for } z=v \\ 0 & \text { for } z \neq v\end{cases}
$$

then

$$
x, y \in V \Rightarrow \delta_{x} \delta_{y}=\varepsilon(x, y) \delta_{x+y} ;
$$

thus, the multiplicative identity of $A(V, \Omega)$ is $1=\delta_{0}$, and if $v \in V$ then $\delta_{v}$ is invertible with $\delta_{v}^{-1}=\delta_{-v}$. In fact (as shown in [8]) the group of units in $A(V, \Omega$ ) is precisely

$$
\left\{\lambda \delta_{v}: v \in V, 0 \neq \lambda \in \mathbb{C}\right\} \text {. }
$$

We shall be interested in subalgebras of $A(V, \Omega)$ generated by units. If $X$ is a subset of $V$, then $A(V, \Omega ; X)$ will denote the subalgebra of $A(V, \Omega)$ generated by $\left\{\delta_{x}: \pm x \in X\right\}$; in addition, $\mathbb{Z} . X, \mathbb{Q} . X$, and $\mathbb{R} . X$ will denote (respectively) the submodules of $V$ generated by $X$ over $\mathbb{Z}, \mathbb{Q}$, and $\mathbb{R}$. We remark that if $X \subset V$ then

$$
A(V, \Omega ; X)=A(V, \Omega ; \mathbb{Z} . X) \text {. }
$$

Glasgow Math. J. 33 (1991) 7-10. 
As a further point of notation, if $X \subset V$ then we define

$$
\begin{aligned}
X^{\perp} & =\{v \in V: \Omega(v, X)=0\}, \\
X^{\prime} & =\{v \in V: \omega(v, X)=1\} ;
\end{aligned}
$$

thus $X^{\perp} \subset X^{\prime}$, with equality when $\mathbb{Q} . X=X$.

It is natural to ask to what extent $A(V, \Omega ; X)$ determines $X \subset V$; the answer to this question is that $A(V, \Omega ; X)$ determines $\mathbb{Z} . X$.

THEOREM. If $S, T$ are additive subgroups of $V$ then

$$
A(V, \Omega ; S) \subset A(V, \Omega ; T) \Leftrightarrow S \subset T .
$$

Proof. We need only demonstrate the forward implication. Let $s \in S$. Since $\delta_{s} \in A(V, \Omega ; T)$ and $T$ is additive,

$$
\delta_{s}=\lambda_{1} \delta_{t_{1}}+\ldots+\lambda_{k} \delta_{t_{k}}
$$

for nonzero $\lambda_{1}, \ldots, \lambda_{k} \in \mathbb{C}$ and distinct $t_{1}, \ldots, t_{k} \in T$. The independence of $\left\{\delta_{v}: v \in V\right\}$ now implies that $k=1, \lambda_{1}=1$, and $s=t_{1}$.

If $B$ is a subalgebra of $A(V, \Omega)$ then $B^{\prime}$ will denote its commutant; note that $B^{\prime}=B$ iff $B$ is maximal abelian.

Theorem. If $X \subset V$ then

$$
A(V, \Omega ; X)^{\prime}=A\left(V, \Omega ; X^{\prime}\right) .
$$

Proof. Let $y \in X^{\prime}$. If $x \in X$ then

$$
\delta_{x} \delta_{y} \delta_{x}^{-1}=\omega(x, y) \delta_{y}=\delta_{y},
$$

so that $\delta_{y}$ commutes with the generators of $A(V, \Omega ; X)$ and so lies in $A(V, \Omega ; X)^{\prime}$. It is now clear that $A\left(V, \Omega ; X^{\prime}\right) \subset A(V, \Omega ; X)^{\prime}$. Let $\phi=\lambda_{1} \delta_{v_{1}}+\ldots+\lambda_{k} \delta_{v_{k}} \in A(V, \Omega ; X)^{\prime}$. If $x \in X$ then

$$
\lambda_{1} \delta_{v_{1}}+\ldots+\lambda_{k} \delta_{v_{k}}=\phi=\delta_{x} \phi \delta_{x}^{-1}=\omega\left(x, v_{1}\right) \lambda_{1} \delta_{v_{1}}+\ldots+\omega\left(x, v_{k}\right) \lambda_{k} \delta_{v_{k}},
$$

so that $\omega\left(x, v_{1}\right)=\ldots=\omega\left(x, v_{k}\right)=1$; thus $v_{1}, \ldots, v_{k} \in X^{\prime}$ and $\phi \in A\left(V, \Omega ; X^{\prime}\right)$. It follows that $A(V, \Omega ; X)^{\prime} \subset A\left(V, \Omega ; X^{\prime}\right)$.

For $X \subset V$ we can now decide when $A(V, \Omega ; X)$ is a maximal abelian subalgebra of $A(V, \Omega)$. Indeed, if $X$ is an additive subgroup of $V$ then

$$
\begin{aligned}
& A(V, \Omega ; X) \text { is maximal abelian } \\
& \text { iff } A(V, \Omega ; X)^{\prime}=A(V, \Omega ; X) \\
& \text { iff } A\left(V, \Omega ; X^{\prime}\right)=A(V, \Omega ; X) \\
& \text { iff } \quad X^{\prime}=X .
\end{aligned}
$$

ThEOREM. If $X$ is an additive subgroup of $V$ then $A(V, \Omega ; X)$ is a maximal abelian subalgebra of $A(V, \Omega)$ iff $X^{\prime}=X$.

In particular, if $F=F^{\perp}$ is a Lagrangian subspace of $(V, \Omega)$ then $A(V, \Omega ; F)$ is a maximal abelian subalgebra of $A(V, \Omega)$. However, $A(V, \Omega)$ has many more maximal 
abelian subalgebras; indeed, it has maximal abelian subalgebras generated by finite sets of units, and it is these that will allow us to extract the dimension of $V$ from the algebraic structure of $A(V, \Omega)$.

Recall that the basis $\left(e_{1}, \ldots, e_{m}, f_{1}, \ldots, f_{m}\right)$ of $V$ is said to be symplectic iff

$$
\Omega\left(e_{i}, e_{j}\right)=\Omega\left(f_{i}, f_{j}\right)=0, \quad \Omega\left(e_{i}, f_{j}\right)=\delta_{i j}
$$

wherever $1 \leq i, j \leq m$.

Theorem. If $X \subset V$ is the integer span of a symplectic basis for $(V, \Omega)$ then $X^{\prime}=X$, so that $A(V, \Omega ; X)$ is a maximal abelian subalgebra of $A(V, \Omega)$.

Proof. Let $X$ be the integer span of the symplectic basis $\left(e_{1}, \ldots, e_{m}, f_{1}, \ldots, f_{m}\right)$. Plainly, $X \subset X^{\prime}$. Let $y \in X^{\prime}$ with

$$
y=\alpha_{1} e_{1}+\ldots+\alpha_{m} e_{m}+\beta_{1} f_{1}+\ldots+\beta_{m} f_{m}
$$

for $\alpha_{i}, \beta_{j} \in \mathbb{R} ;$ then

$$
\alpha_{i}=-\Omega\left(f_{i}, y\right) \in \mathbb{Z}, \quad \beta_{j}=\Omega\left(e_{j}, y\right) \in \mathbb{Z},
$$

so that $y \in X$. Thus, $X^{\prime} \subset X$.

Thus, $A(V, \Omega)$ contains maximal abelian subalgebras generated by $2 m$ units together with their inverses: for example, $A(V, \Omega ; S)$ when $S$ is a symplectic basis of $(V, \Omega)$. Subalgebras of $A(V, \Omega)$ generated by fewer than $2 m$ units and their inverses are never maximal abelian (and of course can fail to be abelian); this will be seen as a consequence of the next result.

Theorem. Let $X=\mathbb{Z} .\left\{x_{1}, \ldots, x_{k}\right\}$ where $x_{1}, \ldots, x_{k} \in V$. If $k<2 m$ then $X^{\perp} \backslash X \neq$ $\varnothing$ so that $X^{\prime} \backslash X \neq \varnothing$.

Proof. Put $Y=\mathbb{R} . X$. The bilinearity of $\Omega$ implies that $Y^{\perp}=X^{\perp}$; moreover,

$$
\operatorname{dim} X^{\perp}=\operatorname{dim} Y^{\perp}=\operatorname{dim} V-\operatorname{dim} Y \geq 2 m-k>0,
$$

so that $X^{\perp} \neq 0$. Since $X$ is countable and $X^{\perp}$ is uncountable, it follows that $X^{\perp} \backslash X$ is uncountable and so nonempty.

THEOREM. If $Z \subset V$ with $\# Z<2 m$ then the subalgebra $A(V, \Omega ; Z)$ of $A(V, \Omega)$ is not maximal abelian.

Proof. From above, if $X=\mathbb{Z} . Z$ then $X^{\prime} \neq X$ and therefore

$$
\begin{aligned}
A(V, \Omega ; Z) & =A(V, \Omega ; X) \neq A\left(V, \Omega ; X^{\prime}\right) \\
& =A(V, \Omega ; X)^{\prime}=A(V, \Omega ; Z)^{\prime},
\end{aligned}
$$

so that $A(V, \Omega ; Z)$ is not maximal abelian.

Gathering these results, we see that if $\operatorname{dim} V=2 m$ then the exponential Weyl algebra $A(V, \Omega)$ contains maximal abelian subalgebras generated by $2 m$ units together with their inverses but does not contain any generated by fewer than $2 m$ units and their inverses. In this way, the dimension of $V$ appears as an algebraic invariant of $A(V, \Omega)$. As promised, we deduce that non-isomorphic symplectic vector spaces have non-isomorphic exponential Weyl algebras. 
TheOREM. Let $\left(V_{1}, \Omega_{1}\right)$ and $\left(V_{2}, \Omega_{2}\right)$ be (finite-dimensional) real symplectic vector spaces. The exponential Weyl algebras $A\left(V_{1}, \Omega_{1}\right)$ and $A\left(V_{2}, \Omega_{2}\right)$ are isomorphic iff $\operatorname{dim} V_{1}=\operatorname{dim} V_{2}$.

In response to the referee's suggestion, we close by indicating how the exponential Weyl algebra is related to other multiplicative analogues of the Weyl algebra. Most closely related is the CCR algebra well-known in mathematical physics: briefly, this is the unique $C^{*}$-algebra completion of $A(V, \Omega)$ as an involutive algebra; see [2, 4]. For any real $\theta$, the rotation algebra $A_{\theta}$ is the $C^{*}$-algebra generated by two unitaries $a$ and $b$ subject to the relation $a b=e^{2 \pi i \theta} b a$; this algebra is studied in [7]. A purely algebraic version of this $C^{*}$-algebra was introduced in [3]: this algebra $B(\lambda, k)$ is generated over the field $k$ by elements $a, b, a^{-1}, b^{-1}$ subject to $a b=\lambda b a$ for some nonzero $\lambda \in k$. A generalization $P(\lambda)$ was studied in [5]: this algebra is generated by units $a_{1}, \ldots, a_{n}$ and their inverses subject to $a_{i} a_{j}=\lambda_{i j} a_{j} a_{i}$ where $\lambda=\left[\lambda_{i j}\right]$ is an $n \times n$ matrix of nonzero elements of $k$. We remark that if $k=\mathbb{C}$ and $|\lambda|=1$ then $B(\lambda, k)$ is a subalgebra of $A(V, \Omega)$ : indeed, it is $A(V, \Omega ; Z)$ where $Z=\{x, y\}$ with $\lambda=\exp \{2 \pi i \Omega(x, y)\}$; moreover, its closure in the CCR algebra is $A_{\theta}$ where $\theta=\Omega(x, y)$. Similarly, $A(V, \Omega)$ contains finitely-generated subalgebras of the form $P(\lambda)$ when $k=\mathbb{C}$ and each $\left|\lambda_{i j}\right|=1$; of course, being uncountably generated, $A(V, \Omega)$ is much larger than any $P(\lambda)$. Finally, we remark that by complexifying the symplectic vector space $(V, \Omega)$ we can lift the restriction that $\lambda$ and each $\lambda_{i j}$ have unit modulus.

ACKnOwledgement. This research was partially supported by the National Science Foundation.

\section{REFERENCES}

1. J. Dixmier, Enveloping algebras (North-Holland, 1977).

2. G. G. Emch, Algebraic methods in statistical mechanics and quantum field theory (Wiley Interscience, 1972).

3. V. A. Jategaonkar, A multiplicative analog of the Weyl algebra, Comm. Algebra 12 (1984), $1669-1688$.

4. J. Manuceau, M. Sirugue, D. Testard, A. Verbeure, The smallest $\mathrm{C}^{*}$-algebra for canonical commutation relations, Comm. Math. Phys. 32 (1973), 231-243.

5. J. C. McConnell and J. J. Pettit, Crossed products and multiplicative analogues of Weyl algebras, J. London Math. Soc. 38 (1988), 47-55.

6. R. Rentschler and P. Gabriel, Sur la dimension des anneaux et ensembles ordonnés, $C . R$. Acad Sci. Paris Sér I Math. 265 (1967), 712-715.

7. M. A. Rieffel, $C^{*}$-algebras associated with irrational rotations, Pacific J. Math. 93 (1981), 415-429.

8. P. L. Robinson, The exponential Weyl algebra, preprint, 1988.

9. J. E. Roos, Propriétés homologiques des quotients primitifs des algèbres enveloppantes des algèbres de Lie semi-simples, C. R. Acad Sci. Paris Sér I Math. 276 (1973), 351-354.

Department of Mathematics

UNIVERSITY OF FLORIDA

GAINESVILLE FL 32611

USA 